ARTICLE

\title{
Harnessing the central dogma for stringent multi-level control of gene expression
}

F. Veronica Greco (10 1, Amir Pandi (10 2, Tobias J. Erb (1) ${ }^{2,3}$, Claire S. Grierson (1) ${ }^{1,4}$ \& Thomas E. Gorochowski (iD ${ }^{1,4 \times}$

Strictly controlled inducible gene expression is crucial when engineering biological systems where even tiny amounts of a protein have a large impact on function or host cell viability. In these cases, leaky protein production must be avoided, but without affecting the achievable range of expression. Here, we demonstrate how the central dogma offers a simple solution to this challenge. By simultaneously regulating transcription and translation, we show how basal expression of an inducible system can be reduced, with little impact on the maximum expression rate. Using this approach, we create several stringent expression systems displaying $>1000$-fold change in their output after induction and show how multi-level regulation can suppress transcriptional noise and create digital-like switches between 'on' and 'off' states. These tools will aid those working with toxic genes or requiring precise regulation and propagation of cellular signals, plus illustrate the value of more diverse regulatory designs for synthetic biology.

\footnotetext{
${ }^{1}$ School of Biological Sciences, University of Bristol, Bristol, UK. ${ }^{2}$ Department of Biochemistry and Synthetic Metabolism, Max Planck Institute for Terrestria Microbiology, Marburg, Germany. ${ }^{3}$ SYNMIKRO Center of Synthetic Microbiology, Marburg, Germany. ${ }^{4}$ BrisSynBio, University of Bristol, Bristol, UK.

$\varpi_{\text {email: }}$ thomas.gorochowski@bristol.ac.uk
} 
S ince the development of the first inducible systems in the early $1980 \mathrm{~s}^{1}$, the ability to dynamically control gene expression through the use of small molecules ${ }^{2}$, light ${ }^{3,4}$, and other signals ${ }^{5}$ has revolutionized biotechnology. From controlling shifts between cell growth and protein production stages during large-scale fermentations ${ }^{6}$, to the detailed characterization of genetic parts and circuitry ${ }^{7}$, the control of gene expression underpins a huge variety of applications. However, while switching expression of a gene 'on' or 'off is conceptually simple, it is rare for genes to have such discrete states or ever be completely silenced. Stochastic effects, 8,9 and leaky expression are widespread and potentially important for adaptation in natural systems but can wreak havoc in engineered systems where genes are toxic to a host or responses are highly sensitive and easily triggered by unavoidable fluctuations ${ }^{10,11}$.

Early systems for controlling gene expression relied on repurposing native regulatory components such as transcription factors. One of the most widely used is the $\mathrm{P}_{t a c}$ system ${ }^{1}$. This consists of a constitutively expressed LacI repressor that can form dimers and tetramers to strongly bind operator sites within a $\mathrm{P}_{\text {tac }}$ promoter sequence and sterically block initiation of RNA polymerase (RNAP). LacI is sensitive to Isopropyl $\beta$-d-1thiogalactopyranoside (IPTG) and at high concentrations, the DNA binding activity of LacI is abolished. This lifts repression of $\mathrm{P}_{\text {tac }}$ and leads to strong transcription of genes regulated by this promoter. While in most cases such systems offer strong repression, because such regulatory systems focus on a single step during protein synthesis (i.e. transcription), they are vulnerable to fluctuations in regulator production and the stochastic nature of biochemical reactions during gene expression ${ }^{9}$.

Over the past decade, synthetic biologists have developed more advanced methods to control gene expression. These include engineered regulators based on DNA binding proteins, such as zinc fingers ${ }^{12}$, TALENs ${ }^{13}$ and CRISPRi ${ }^{14}$, RNA-RNA interactions ${ }^{15-17}$, post-transcriptional/translational processes such as RNA and protein degradation ${ }^{18}$, as well as using directed evolution to optimize existing inducible systems ${ }^{19}$. This offers a wealth of options to more strictly regulate gene expression through the coupling of multiple forms of regulation (e.g. affecting both transcription and translation of a gene) to reduce unwanted expression and improve the robustness of a system to component failure. However, few examples of such multi-level regulation have been implemented to date $^{20,21}$. This has resulted in an unclear picture of how best stringent multi-level control can be achieved and the trade-offs that exist between performance, regulatory complexity, and cellular burden when designing these systems.

Here, we address this problem by systematically studying the combined use of transcriptional and translational regulators to stringently control protein expression. Using a combination of mathematical modelling and combinatorial genetic assembly, we are able to design, build and test a variety of synthetic multi-level controllers (MLCs) and elucidate the relative performance of each. These controllers all implement a coherent type 1 feedforward loop (C1-FFL) regulatory motif (Fig. 1a) that is commonly found in natural genetic systems and is known to enable more stringent control of an output but is rarely used when designing new expression systems ${ }^{22}$. We show how MLCs offer advantages for many applications spanning the stringent control of protein expression to the accurate propagation of information in a cell ${ }^{23,24}$ and demonstrate how applying modern synthetic biology tools to even simple regulatory systems can offer paths towards the precise and reliable control of biological systems.

\section{Results}

Stringent control of gene expression by harnessing the central dogma. In most synthetic genetic circuits, control of gene expression is achieved through the use of a single type of regulation (Fig. 1a), with control of transcription predominantly used. While this type of single-level controller (SLC; Fig. 1b) is often sufficient for many applications, the central dogma naturally lends itself to more stringent multi-level regulation where both transcription and translation are controlled simultaneously (e.g. via transcription factors and RNA-based translational switches). Such multi-level controllers (MLCs; Fig. 1c) can be generalised by a genetic design that consists of an $L 1$ gene encoding a level 1 transcriptional regulator with cognate promoter $\mathrm{P}_{L 1}$, and an $L 2$ gene encoding a level 2 translational regulator. Both $L 2$ and the gene of interest (GOI) are separately transcribed by $\mathrm{P}_{L 1}$ promoters and the product of $L 2$ activates translation of the GOI transcript. This MLC encapsulates a coherent type 1 feed-forward loop (C1-FFL) in which both $L 1$ and $L 2$ are necessary for production of the GOI.

To explore the possible benefits of this regulatory motif, we developed mathematical models to capture how the rate of production of a GOI varied in response to differing concentrations of an input inducer for both the SLC and MLC designs (Supplementary Note 1; Supplementary Data 1). We generated steady-state response functions by simulating the models using biologically realistic parameters (Supplementary Table 1) over a range of different input IPTG concentrations. As expected, the output production rate displayed a sigmoidal shape with both controllers reaching near identical maximum rates at high input IPTG concentrations (Fig. 1d). The main difference was that the MLC design displayed a 50-fold lower output than the SLC design at low IPTG concentrations, leading to significantly reduced basal expression when no input was present (Fig. 1d). This caused the MLC design to have both an increased dynamic range and fold change between 'off' and 'on' states when compared to the SLC design.

We also simulated the output protein production rate for both models when exposed to a range of dynamic inputs. These included delta functions, as well as pulse and step inputs (Fig. 1e). Simulations showed that both types of controller displayed virtually identical output responses for both the pulse and step inputs, with only a small reduction in output expression rate for the MLC that matched its lower basal expression level. However, significant differences were observed in the responses to the delta function input. While the SLC led to moderate sized pulses in output, the MLC design fully suppressed all output activity with only tiny fluctuations in the output expression rate observed. The behaviour of the MLC arose from the need for both $L 1$ and $L 2$ to be expressed to sufficiently high levels for expression of the GOI to be triggered. The short pulses of expression caused by the delta function input were insufficient to cause this switch and allowed the MLC to effectively filter out these transient events in its input.

The ability to filter out rapid fluctuations is particularly important for stringent control in systems where input promoters exhibit high levels of intrinsic noise. In such scenarios, protein levels can vary significantly across a population of cells $s^{8}$ due to the often bursty nature of gene transcription. This is commonly seen for weak promoters where intrinsic noise dominates. Rather than the activity of a weak promoter being uniformly low, it instead displays short bursts of strong activity separated by long periods of inactivity 8,25 . Across a population this averages out to a low overall expression level, but large variability is present between cells. As seen for the delta function inputs, such input profiles driving the SLC will lead to large fluctuations in the output. However, because intrinsic promoter noise is specific to an individual promoter and uncorrelated between multiple identical versions of a promoter within a construct, the MLC design, which contains two copies of the input promoter $\mathrm{P}_{L 1}$, should find that a burst of expression from one $\mathrm{P}_{L 1}$ promoter 


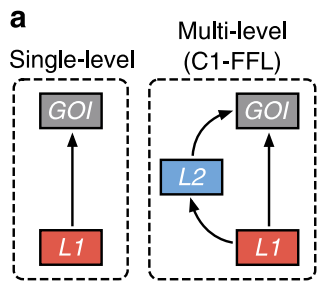

b
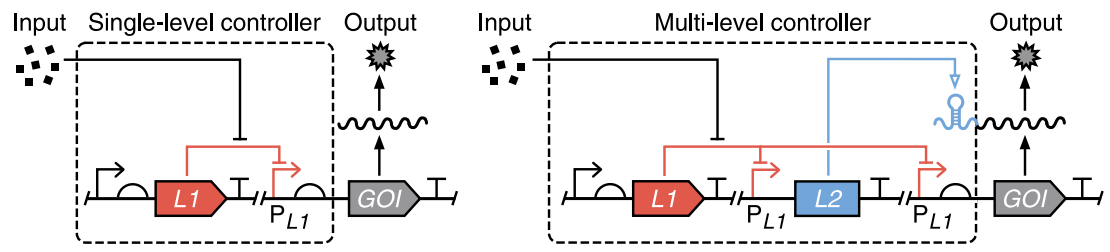

d

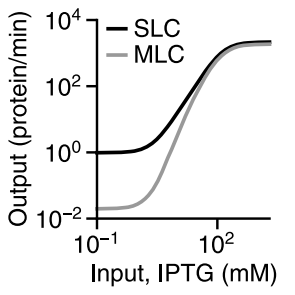

e

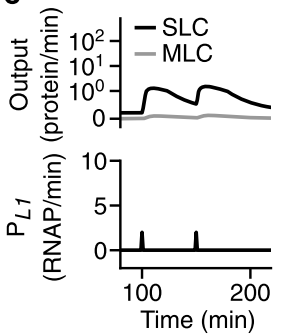

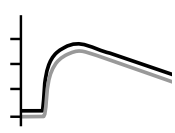
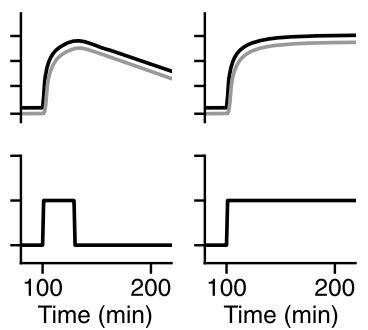

$\mathbf{f}$

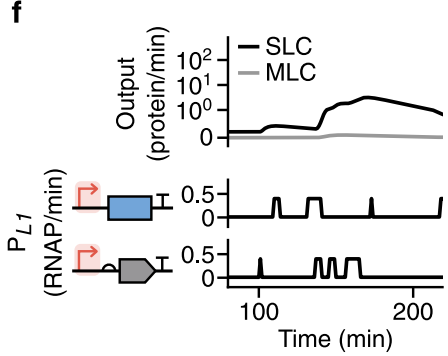

Fig. 1 Stringent control of protein expression through multi-level gene regulation. a Two possible regulatory schemes to control the expression of a gene of interest (GOI): (1) control using a single regulator (L1), and (2) multi-level control using two separate regulators (L1 and $L 2)$ connected in the form of a coherent type 1 feed-forward loop (C1-FFL). b Schematic of a genetic implementation of a single-level controller (SLC) that uses only transcriptional (red lines) regulation. An input (e.g. small molecule) modulates activity of the $\mathrm{P}_{L 1}$ promoter and production of the GOI. c Schematic of a genetic implementation of a multi-level controller (MLC) that uses both transcriptional (red lines) and translational (blue line) regulation. An input (e.g. small molecule) modulates activity of the two $P_{L 1}$ promoters and an internal $L 2$ regulator activates the translation of $G O I$ transcripts to finally produce the output protein. $\mathbf{d}$ Steadystate response functions from mathematical models of the SLC and MLC. e Dynamic model simulations of the SLC and MLC and their response to different forms of temporal input (left to right): delta functions $\left(P_{L 1}\right.$ activity $=2$ RNAP/min for 1 min at $100 \mathrm{~min}$ and $\left.150 \mathrm{~min}\right)$, a pulse $\left(P_{L 1}\right.$ activity $=5 \mathrm{RNAP} / \mathrm{min}$ from 100-130 min), and a step function ( $P_{L 1}$ activity $=5$ RNAP/min from 100 min onwards). The activity of both $P_{L 1}$ promoters in the MLC is considered identical. $\mathbf{f}$ Dynamic model simulations of the SLC and MLC showing suppression of intrinsic promoter noise by the MLC. The two identical $P_{L 1}$ promoters for the $L 2$ regulator and $\mathrm{GOI}$ are separately driven by independent and biologically realistic bursty transcriptional activity profiles (Methods).

is highly unlikely to occur at the same time as a burst from the other. Therefore, the MLC will suppress noise in the output.

To test this hypothesis, we generated accurate time-series promoter activity profiles based on a two-state model ${ }^{25}$ where the mean length of time a promoter was in an 'on' active and 'off silent state $\left(\Delta t_{\mathrm{ON}}\right.$ and $\Delta t_{\mathrm{OFF}}$, respectively) were $\left\langle\Delta t_{\mathrm{ON}}\right\rangle=6 \mathrm{~min}$ and $\left\langle\Delta t_{\mathrm{OFF}}\right\rangle=37 \mathrm{~min}$. These values were taken from previous experimental measurements in $E$. coli ${ }^{25}$. We also set the activity of the $\mathrm{P}_{L 1}$ promoter when in an 'on' state to a biologically realistic $0.25 \mathrm{RNAP} / \mathrm{min}$. Independent time-series were generated for each $\mathrm{P}_{L 1}$ promoter in the MLC and only one of these was used for the SLC where only a single $\mathrm{P}_{L 1}$ promoter is present. These profiles were then fed into our existing dynamic models and the responses of the systems simulated. We found that the output production rate for the SLC saw large increases, especially where the input consisted of longer bursts of activity or several bursts in short succession (Fig. 1f). In comparison, the MLC fully suppressed all output production making it an excellent filter of intrinsic promoter noise.

A genetic template to explore multi-level gene regulation. There are many ways that an MLC could be implemented biologically. Furthermore, when implementing such a controller it is often necessary to switch the input that is used and internal regulators such that multiple controllers can be used simultaneously within the same cell. To meet these requirements, we developed an 8-part genetic template and toolkit of parts to allow for the rapid combinatorial assembly of MLCs (Fig. 2a). The design enables both single and multi-level regulation, has the option to introduce protein tags for further post-translational control of the GOI (e.g. through protein degradation) and is structured to minimise the chance of transcriptional readthrough that would cause unwanted expression of the component parts. The toolkit comprises eight types of part plasmid ( $\mathrm{pA}-\mathrm{pH})$ and a backbone plasmid (pMLC-BB1) in which the final MLC design is inserted (Supplementary Data 2). Assembly is performed using a standard one-pot Golden Gate reaction with individual blocks designed to use 4-bp overhangs with minimal cross reactivity to ensure the correct and efficient ligation of parts ${ }^{26}$. Furthermore, rapid screening of successful inserts is enabled by the drop-out of an orange fluorescent protein (ofp) expression unit ${ }^{27}$ (Supplementary Note 2).

Using this toolkit, we aimed to compare the in vivo behaviours of different SLC and MLC designs with a focus on the different mechanisms that could be used for $L 2$ control and the affect these might have on overall performance. For the SLC design we chose the widely used $\mathrm{P}_{t a c}$ system introduced earlier (Fig. 2c). To simplify comparisons, we also used the $\mathrm{P}_{t a c}$ system for $L 1$ control in all the MLC designs and combined it with three different RNAbased $L 2$ regulators. These included a toehold switch (THS; Fig. 2d) ${ }^{17,28}$, a small transcription activating RNA (STAR; Fig. 2e) ${ }^{29}$, and a dual control system (DC; Fig. $\left.2 \mathrm{f}\right)^{21}$.

The THS regulator encodes a structural component followed by a ribosome binding site (RBS) that is used to drive translation of the GOI (Fig. 2d). The structural region is designed to form a strong hairpin loop that when transcribed hinders the ability for ribosomes to bind the RBS, and thus inhibits translation. Translation is activated by expression of a complementary small RNA (sRNA) trigger that hybridizes to a short unstructured region of the THS, causing a breakdown in its secondary structure. This conformational change allows ribosomes to bind the RBS and translation of the GOI to proceed. THSs were selected because they offer strong repression of translation, can be designed computationally, and large libraries of designs exist with minimal crosstalk when used together ${ }^{17,28}$.

Unlike the THS, the STAR regulator works at a transcriptional level. The STAR's target is placed before an RBS in the $5^{\prime}$ untranslated region (UTR) of the GOI (Fig. 2e). This forms an 

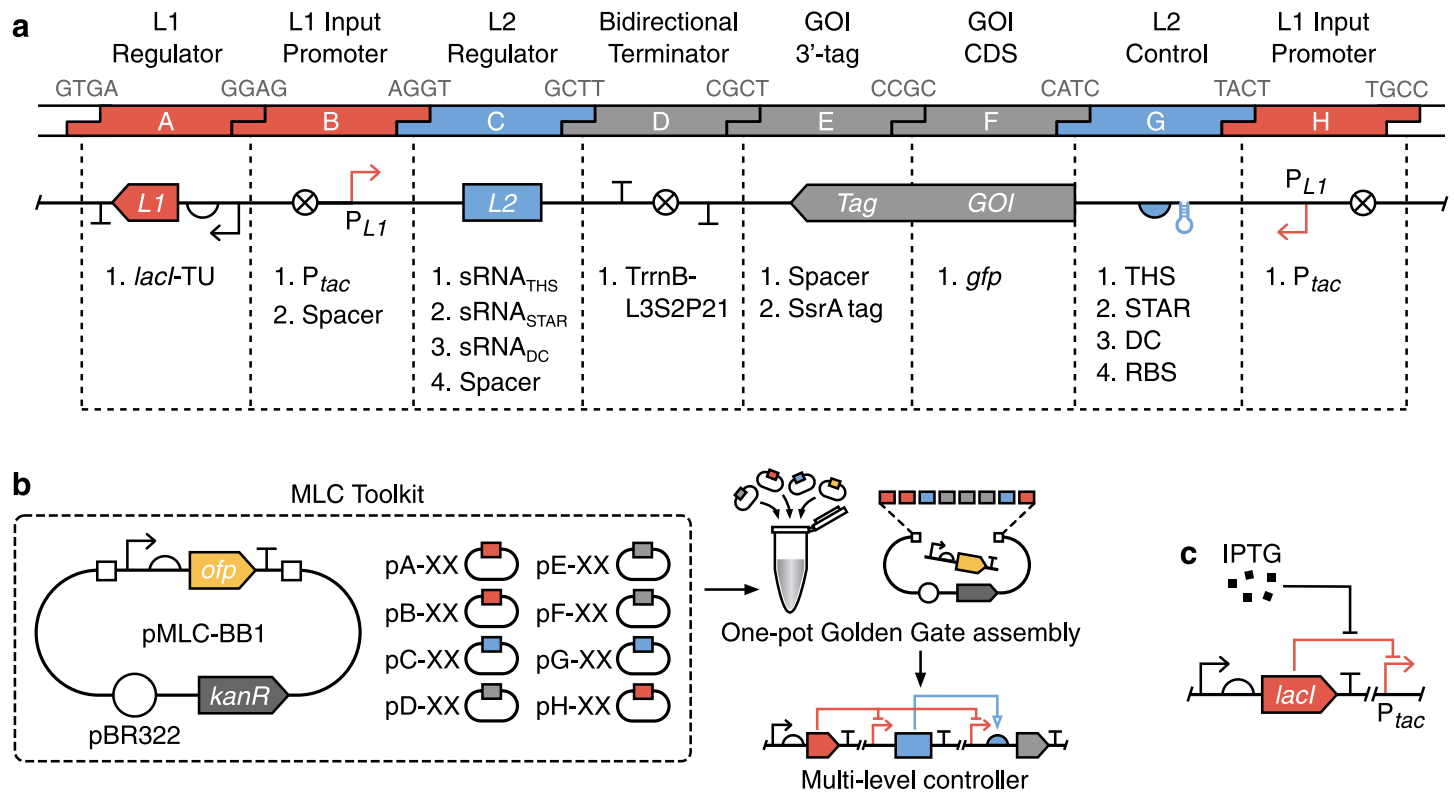

Multi-level controlle
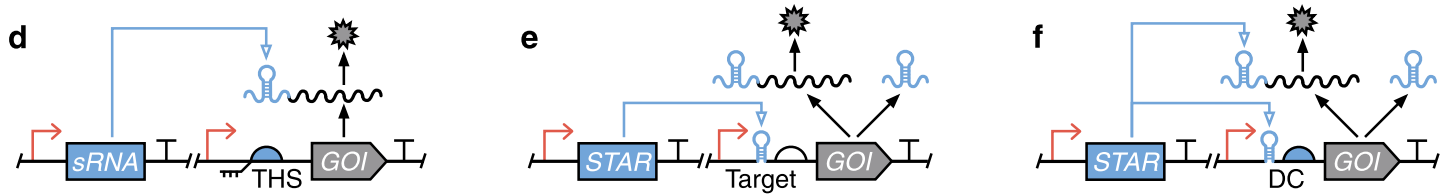

Fig. 2 Combinatorial assembly of gene expression controllers. a Summary of the 8-part genetic template used to allow for systematic exploration of direct and multi-level gene regulation. The 4-bp overhangs used for Golden Gate assembly are shown in grey at their respective junctions. Available genetic elements are listed below each corresponding part type $(\mathrm{A}-\mathrm{H})$. b The MLC toolkit contains a set of plasmids that can be combined using Golden Gate assembly to create a variety of direct and multi-level controllers (Supplementary Fig. 3). c The lacl transcription factor responsive to IPTG used for level 1 (L1) transcriptional regulatory control. d Toehold switch (THS) translational regulator used for level 2 (L2) control. e Small transcription activating RNA (STAR) transcriptional regulator used for $L 2$ control. $\mathbf{f}$ Dual control (DC) transcriptional and translational regulator used for $L 2$ control.

intrinsic terminator when transcribed and inhibits GOI expression. Activation is achieved by expression of the STAR RNA, which interacts with the target, prevents terminator formation and thus allows for expression of the downstream GOI. Similar to THSs, STARs have been shown to offer strong repression and there exist large libraries of orthogonal variants ${ }^{16,29}$.

Finally, the DC regulator combines both transcriptional and translational control by modifying the pT181 attenuator ${ }^{21}$. The DC target is placed in the $5^{\prime}$ UTR of the GOI and encodes an intrinsic terminator that includes the RBS (Fig. 2f). When transcribed, the intrinsic terminator not only halts transcription, but also represses translation by causing the RBS to form a strong RNA secondary structure making it inaccessible to the ribosome. Activation is achieved by expression of a STAR, which interacts with the target, both preventing terminator formation and causing a conformational change in the RNA structure that makes the RBS accessible for translation initiation. The DC regulator was chosen due to this combined regulatory action, which has been shown to produce strong repression ${ }^{21}$. However, to date, only a single regulator like this has been created, limiting future applications.

DNA encoding parts for each of these regulatory systems were synthesised and our toolkit used to assemble the SLC and three MLC designs. Superfolder green fluorescent protein (GFP) was chosen as the GOI to allow for the measurement of output expression in single cells using flow cytometry.

Performance comparison of the controllers. To characterise the performances of the controllers, we transformed Escherichia coli cells with each construct and measured GFP fluorescence using flow cytometry for 'off and 'on' input states. As $\mathrm{P}_{\text {tac }}$ was used as an input for all the designs, this corresponded to growing the cells in either 0 or $1 \mathrm{mM}$ IPTG, respectively (Methods). Data from these experiments was then used to calculate the dynamic range and fold change in output GFP fluorescence (Table 1; Supplementary Fig. 1).

We found a clear separation between output states for all designs with little variation between biological replicates (Fig. 3a). All MLCs (THS, STAR, and DC) reached higher expression levels than the $\mathrm{P}_{t a c}$ SLC, and the THS and DC designs achieved large $>1000$-fold changes between output states. Notably, while the STAR design reached a much higher 'on' state than the $\mathrm{P}_{t a c}$ design, the STARs high levels of basal (leaky) expression when no input was present resulted in a $43 \%$ lower fold change (Table 1).

A challenge when calculating these measures (especially fold change) is the ability to accurately quantify very low levels of output GFP fluorescence, which are near or identical to the autofluorescence of the cells. To better understand this aspect, we measured the GFP autofluorescence of untransformed E. coli cells, performing 11 biological replicates to estimate a fluorescence distribution that could be used as an approximate detection limit. Overlaying the average and standard deviation of the cell autofluorescence onto our results (Fig. 3a, dashed line and grey shaded region), we found that the 'off states for the $\mathrm{P}_{t a c}$, THS, and DC designs all fell within this region and very close to the average suggesting they have virtually no leaky expression at all.

Another difficulty when comparing the performance of the controllers is the need to consider the large differences in the 
Table 1 Performance summary of the single- and multi-level controllers in vivoa.

\begin{tabular}{|c|c|c|c|c|c|c|}
\hline Controller & Type $^{b}$ & Basal'c (\%) & Dynamic range $^{d}\left(10^{3}\right.$ MEFL) & Fold change $^{d}$ & Co-operativitye, $n$ & $S N R^{f}(d B)$ \\
\hline$\overline{P_{t a c}}$ & SLC & 0.5 & 0.9 & 93 & 3.4 & 0.2 \\
\hline THS & MLC & 0.02 & 65.5 & 2166 & 7.3 & 10.1 \\
\hline STAR & MLC & 1.45 & 4.6 & 53 & 9.3 & 4.7 \\
\hline $\mathrm{STAR}_{2}$ & MLC & 2.0 & 3.4 & 37 & 7.7 & 4.5 \\
\hline DC & MLC & 0.04 & 10.4 & 1030 & 3.4 & 7.1 \\
\hline
\end{tabular}

aAll values are averages calculated from three biological replicates. Key performance features of the controllers are visually shown in Supplementary Fig. 1.

bSLC refers to 'single-level controller' and MLC refers to 'multi-level controller'.

'Relative basal expression calculated when no IPTG is present and as a percentage of the expression level for the 'on' state (1 mM IPTG).

'Calculated between 'on' and 'off' states for cells grown in 0 and $1 \mathrm{mM}$ IPTG, respectively, and given in calibrated molecules of equivalent fluorescein (MEFL) units.

eFrom the Hill function fitting of the steady-state response functions (Fig. 4a).

'SNR refers to 'Signal to Noise Ratio'.
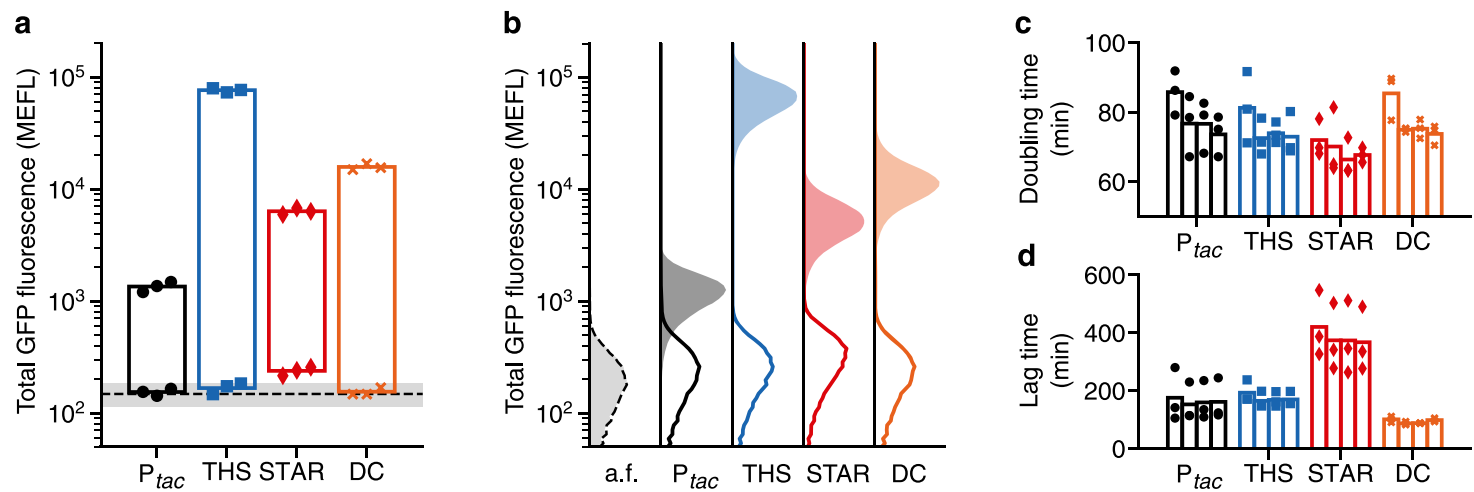

Fig. 3 Performance comparison of single- and multi-level controllers in vivo. a Total GFP fluorescence for 'off' and 'on' input states ( 0 and $1 \mathrm{mM} \mathrm{IPTG,}$ respectively). Points show the three biological replicates for each controller and condition (black circles, $\mathrm{P}_{\text {taci }}$ blue squares, THS; red diamonds, STAR; orange crosses, DC). Black dashed line denotes the mean fluorescence of cell autofluorescence (a.f.) controls containing no plasmid with grey shaded region showing \pm 1 standard deviation of 11 biological replicates. Fluorescence given in calibrated molecules of equivalent fluorescein (MEFL) units. $\mathbf{b}$ Flow cytometry distributions of total GFP fluorescence for 'off' (line) and 'on' (shaded) input states. Cell autofluorescence (a.f.) controls containing no controller are shown by black dashed line and light grey filled distributions. c Doubling time of cells harbouring direct and multi-level controllers for varying concentrations of IPTG (bars left to right for each design: 0, 0.1, 1, $10 \mathrm{mM} \mathrm{IPTG).} \mathbf{d}$ Lag time calculated as the time to reach an $\mathrm{OD}_{600}=0.15$ after inoculation of cells harbouring controllers for varying concentrations of IPTG (bars left to right for each design: 0, 0.1, 1, 10 mM IPTG). Source data are provided as a Source Data file.

maximum expression rates (e.g. $>60$-fold difference between the $\mathrm{P}_{t a c}$ and THS designs for the 'on' state). It should be noted that the same $\mathrm{P}_{\text {tac }}$ promoter is used as input to all our designs and that it includes a 15-bp upstream spacer element to insulate its function from contextual effects arising from differing nearby sequences that are present in each design ${ }^{24,30}$. It is therefore reasonable to expect the dynamic range of the input promoter's transcriptional activity to be similar for each controller, with differences in output protein expression rate related directly to the different strength ribosome binding sites found in each L2 regulator or the SLC design. Given these differences and to allow for an unbiased comparison, we calculated the relative basal GFP expression level of each controller as a percentage of its maximum output (Table 1). This showed that the THS performed best, displaying a 25-fold decrease in relative basal expression compared to the $\mathrm{P}_{t a c}$ SLC with $0.02 \%$ relative basal expression compared to $0.5 \%$, respectively. The DC design also performed well with $0.04 \%$ relative basal expression, while the STAR MLC saw the largest relative basal expression of $1.45 \%$, nearly three times that of the $\mathrm{P}_{t a c}$ SLC.

While comparisons of average expression levels between 'on' and 'off states are useful, they are not able to capture the role of cell-to-cell variability inherent in all gene expression ${ }^{9}$. Such variation is crucial when assessing the performance of stringent expression systems because even though average output states might be sufficiently separated to be distinguished, cell-to-cell variation across a population can lead to overlaps in the output distributions. Cells falling in this overlap are impossible to classify resulting in some cells with an undetermined state. Engineers have developed measures to help characterise the strength and quality of a signal (i.e. the ability to distinguish 'on' and 'off' output states) with the Signal to Noise Ratio (SNR) commonly used in other fields such as electronics. SNR has also recently been adapted for use when studying engineered genetic systems making it easier to understand how the quality of signals in a circuit are maintained or degraded as they pass through various genetic devices $^{23}$.

Using the flow cytometry distributions, we calculated the SNR for each controller in decibel (dB) units (Table 1; Methods). We found that the $\mathrm{P}_{t a c}$ SLC performed worst with a low SNR of 0.2 $\mathrm{dB}$, corresponding to a signal barely larger than the noise. This was evident for the flow cytometry distributions where a sizable overlap in the 'on' and 'off states was seen (Fig. 3b). All MLCs performed better with the THS achieving an SNR $>10 \mathrm{~dB}$. This improved performance was also evident from the flow cytometry data with clear gaps of varying sizes between the 'on' and 'off output distributions (Fig. 3b). This clearer separation between cells in an 'on' and 'off state would make these parts ideal for genetic logic circuits, ensuring signals are cleanly propagated.

Burden of controllers on the host cell. There is a growing awareness of the importance of considering the burden that engineered genetic parts and circuits place on their host cell ${ }^{31}$. The introduction of a genetic construct that sequesters large 

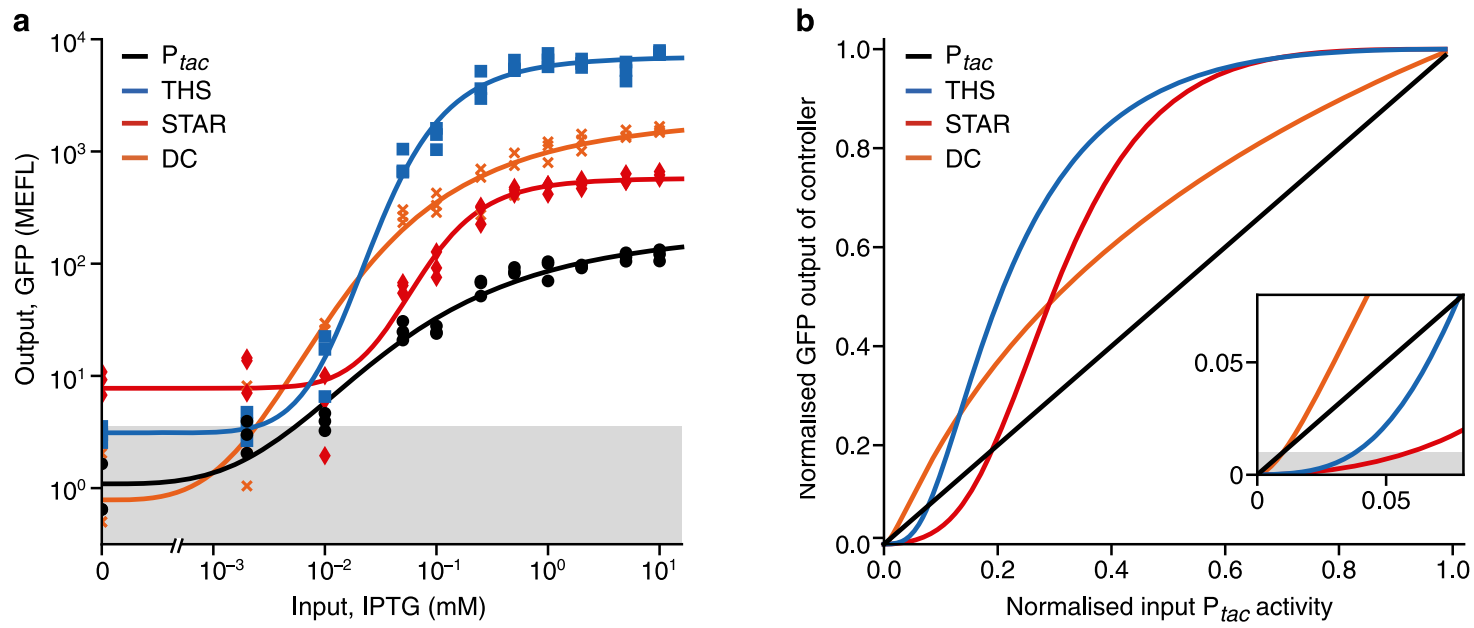

Fig. 4 Response functions of single- and multi-level controllers in vivo. a Steady-state response functions of the controllers showing output GFP fluorescence (corrected for cell autofluorescence) for varying input IPTG concentrations (0, 0.002, 0.01, 0.05, 0.1, 0.25, 0.5, 1, 2, 5, 10 mM). Points show the three biological replicates for each controller and condition (black circles, $P_{\text {taci }}$ blue squares, THS; red diamonds, STAR; orange crosses, DC). Grey shaded region shows the standard deviation of cellular GFP autofluorescence from 11 biological replicates. b Comparison of how normalised GFP output (as a fraction of the maximum GFP fluorescence) varies in response to changes in the normalised transcriptional activity of $P_{\text {tac }}$ (as a fraction of its maximum activity). Multi-level regulation can lead to the suppression or amplification of the output GFP production rate compared to direct transcriptional regulation (i.e. a specific multi-level controller's line falls below or above the diagonal, respectively). Insert shows zoomed area and grey shaded region denotes a GFP output level of $1 \%$ for the controller. Source data are provided as a Source Data file.

quantities of shared cellular resources like ribosomes or heavily impacts core metabolic fluxes can lead to reduced growth rates and trigger stress responses that impair the function of engineered genetic parts ${ }^{32-37}$. When designing the MLCs, we purposefully selected RNA-based regulators as previous results suggest that they impose a small metabolic burden on the cell ${ }^{38}$. To experimentally verify this in our cells, we generated growth curves for all SLC and MLC designs (Supplementary Fig. 2). Because the metabolic demands of the controllers would vary based on the concentration of inducer present (due to the varying levels of sRNA or STAR produced), cells were exposed to four different concentrations of IPTG $(0,0.1,1,10 \mathrm{mM})$ spanning the 'off and 'on' states of the controllers.

From these growth curves, we estimated the doubling time during the exponential growth phase (Methods). We found that the SLC and all MLCs displayed similar doubling times of $\sim 70$ min (Fig. 3c). Furthermore, we saw a slight decrease in the doubling times of all controllers as the IPTG concentration increased. This trend is counterintuitive given that an increasing IPTG concentrations will cause expression of the GOI and any L2 regulators, increasing the burden on the cell. However, it is known that IPTG can have unexpected effects on cell physiology ${ }^{39}$ and cause changes in plasmid stability ${ }^{40}$, which could lead to reduced overall burden due to fewer copies of the controller plasmid or more efficient utilisation of available nutrients by the cell.

We also measured the lag time after inoculation into fresh media before the cells entered exponential growth (Methods). We found differences between many of the controllers with a lag time of $\sim 165 \mathrm{~min}$ for the $\mathrm{P}_{t a c}$ and THS designs, a shorter lag time of 88 min for the DC design, and a significantly longer lag time of 373 min for the STAR design (Fig. 3d). Closer inspection of the growth curves showed that the DC design had a consistently higher initial cell density (optical density at $600 \mathrm{~nm}$ of 0.07 compared to 0.04 for the THS design), which could account for the shorter lag phase (Supplementary Fig. 2). For the STAR design the elongated lag phase coincided with a consistently longer additional time of $\sim 100 \mathrm{~min}$ to reach saturation of the culture.
To better understand if the extended lag phase of the STARbased MLC was a general feature to be expected when using this type of regulator, we rebuilt this construct using a different STAR $\left(\mathrm{STAR}_{2}\right)$ that had an identical initial $72 \mathrm{bp}$ sequence, but unique 10 bp sequence at its $3^{\prime}$-end (Supplementary Table 2). As we would expect for such a similar design, testing of the $\mathrm{STAR}_{2}$ construct showed similar performance to the initial STAR design with a good dynamic range and similar leaky expression in its output (Table 1; Supplementary Fig. 3; Methods). However, unlike the original, the STAR ${ }_{2}$ design displayed a lag phase (161 $\mathrm{min}$ ) and doubling time (72 $\mathrm{min})$ that closely matched the other MLCs. This suggests that the long lag times observed for the original STAR design were likely due to some highly specific and uncharacterised off-target interactions with endogenous cellular processes and not due to a general feature of the STAR's regulatory mechanism.

Digital-like transitions and suppression of weak input signals. Our previous modelling of the MLCs showed that in addition to improved performance in 'on' and 'off' states, the addition of the L2 regulator also altered the response function, causing a sharper transition from an 'off to an 'on' state due to the lower relative basal expression, and an ability to suppress low-level noise in the input (Fig. 1d, e).

To assess if these features were present, we generated response functions of the controllers by growing the cells in varying concentrations of input inducer and measuring steady-state output GFP fluorescence. The sharpness of the transition is captured by the co-operativity of Hill function fits to this data. We found that in comparison to the $\mathrm{P}_{t a c}$ SLC, both the THS and STAR MLCs saw more than a doubling in this value from 3.4 to more than 7, while the DC design maintained an identical value (Table 1). High cooperativities correspond to a very sharp steplike transition between 'on' and 'off states that is clearly evident from the response function curves (Fig. 4a). The high nonlinearity in the response functions of the THS and STAR MLCs is potentially useful for information processing tasks. In particular, implementing digital logic within cells requires clear 'on' and 'off states and limited chance for signals to reside at 
intermediate states. Sharp transitions in the response function ensure that there is less room for an input to fall at an intermediate point during the transition, ensuring an 'on' or 'off state is always given. Furthermore, a high nonlinearity can also be exploited to generate bimodality. For example, if a noisy input is positioned to span the transition point in the response function, a population of cells will have large groups of cells in 'on' and 'off' states, with much fewer in intermediate states because of the sharp transition and small probability of falling in this small region.

To quantify the ability of each MLC to suppress low-level input noise, we further analysed the response functions. As mentioned earlier, the large differences in dynamic range make comparisons between designs difficult. Given that the promoter driving transcription for each MLC is identical $\left(\mathrm{P}_{t a c}\right)$, the discrepancies arise from differing $g f p$ translation rates controlled by the associated ribosome binding sites. These do differ in sequence and strength for each design and in some cases are specific and integral to the RNA regulator's function. Therefore, to allow for comparisons, we normalised the output of each MLC to its maximum output and used data from the $\mathrm{P}_{t a c}$ SLC to estimate the input activity of the $\mathrm{P}_{t a c}$ promoter used in each controller. If no secondary regulation was present (as in the SLC), then we would expect the normalised input and output to follow a straight line where one equals the other (see $\mathrm{P}_{t a c}$ design in Fig. $4 \mathrm{~b}$ ). However, if the secondary regulation suppresses the input $\mathrm{P}_{t a c}$ activity then a lower normalised output to input will be seen, and conversely, an amplification of the input will lead to a higher normalised output to input.

Using this approach, we assessed the responses of each MLC and found that all caused a suppression of low levels of input promoter activity and an amplification of higher input activities. This effect was most prominent for the THS and STAR designs, with both able to ensure controller output is maintained below $1 \%$ even when the input promoter reaches $3.5 \%$ activity (Fig. $4 \mathrm{~b}$, insert). These results confirm the findings of our modelling and demonstrate the potential for using MLCs to filter out unwanted input activity in noisy environments.

Controller performance in a cell-free expression system. There has been growing interest in the use of cell-free protein synthesis (CFPS) systems ${ }^{41}$ as a means to prototype synthetic genetic circuits $^{42}$, enable the rapid characterisation of genetic parts and metabolic pathways ${ }^{43}$, and more recently as a novel bioproduction platform ${ }^{44}$. While great progress has been made in expanding the applications of CFPS systems ${ }^{45-48}$, strategies to stringently control protein expression have yet to be developed.

To assess the performance of our controllers in a cell-free context, we used a CFPS system created from crude E. coli cell lysate and performed simple batch reactions (Methods) that we continuously monitored so that output expression rate could be inferred from changes in GFP fluorescence over time. These experiments showed that all controllers were also functional in the CFPS system and behaved qualitatively similar to the in vivo situation (Fig. 5a; Supplementary Table 3). Overall, MLC designs performed better than the SLC design by showing lower percentages of basal expression, larger dynamic ranges and larger fold changes between 'off and 'on' states with sharper, more digital-like, transitions (i.e. higher co-operativity in Hill function fits).

However, compared to the in vivo situation, we observed distinct differences in performance. The largest drop in performance was observed for the $\mathrm{P}_{t a c}$ SLC design, for which basal expression reached $10 \%$ of the maximal output and only a 10 -fold dynamic range (i.e. between 'off and 'on' output states).
Performance losses were also observed for the other MLC designs. However, the THS design displayed $<1 \%$ relative basal expression and a $\sim 350$-fold change between 'off and 'on' output states. These performance losses were likely caused by the relatively low effective concentrations of regulators that can be achieved in a CFPS system compared to the highly crowded cytoplasm of a living cell ${ }^{49}$. Especially low concentrations of the LacI repressor will likely limit the maximal repression that can be achieved in the CFPS system, accounting for the higher basal expression observed.

Notably, the STAR MLC showed a similar performance in respect to percentage of basal expression, fold change and cooperativity compared to the in vivo setting (Table 1 and Supplementary Table S3). This robustness may stem from the fact that the STAR design exploits secondary control at a transcriptional level, specifically, through premature termination of transcription in the $5^{\prime}$ UTR of the output gene, and therefore regulation limits the potentially active transcripts that are present within the reaction (Fig. 2e). In contrast, the THS design produces full length transcripts and relies on continuous suppression of translation initiation by RNA secondary structures (Fig. 2d). Our data suggest that the STAR regulator is less affected by the differing environment of the CFPS system than the THS, enabling the STAR design to maintain virtually identical performance across these contexts.

Time-course measurements from these experiments also allowed us to quantify the output GFP production rates as the reactions proceeded. This data revealed a key difference between the in vivo and CFPS system that was observed for all controller designs. For the first 2 hr the expression rates for 'off and 'on' states for each design were virtually identical, with regulation only being observed after this point and strongly affecting output GFP production rate after $4 \mathrm{hr}$ (Fig. 5b). The initial constant output GFP production rates in the CFPS system matched the order of different RBS strengths measured in vivo. The more rapid decrease in expression observed for the MLC designs versus the SLC for the regulated 'off state is expected because of the additional regulatory layer ( $L 2$ regulator) of these designs. The observed 'lag' phase of the regulation very likely reflects the time required for each controller to express sufficient LacI to interact with the $\mathrm{P}_{t a c}$ promoters that act as the input in all our designs. In contrast to the CFPS system, cells in the in vivo experiments were in exponential growth and the MLCs were likely at steady state with LacI degradation and dilution rates equalling its production rate which ensured a constast concentration of repressor. Therefore, while multi-level regulation offers greatly improved control over gene expression in CFPS systems, for batch reactions, it is crucial that necessary regulatory components (e.g. repressor proteins) are present at sufficient concentrations from the start of an experiment to enable stringent regulation. This could be achieved by generating the CFPS system from cells that already express the regulators at high concentrations, by separately adding these components into the reaction mix before an experiment starts, or by making use of microreactors to enable the CFPS system to maintain steady-state concentrations of regulators through continual dilution of the reaction products ${ }^{50}$.

\section{Discussion}

In this work we have shown how multi-level control of gene expression offers a means to more stringently regulate gene expression both in vivo and in vitro. By harnessing the multi-step process of transcription and translation that underpins the central dogma of biology and simultaneously regulating both processes in response to an input signal, we demonstrate through modelling (Fig. 1) and experiments (Figs. 3-5) how inducible expression 
a

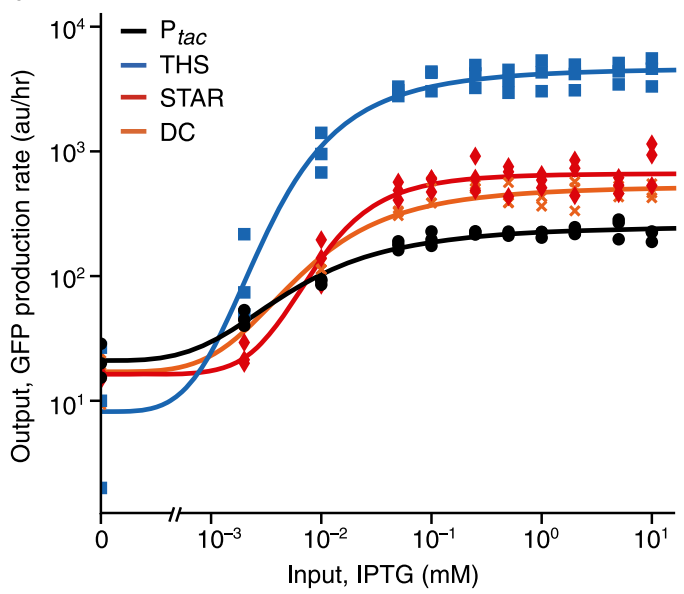

b

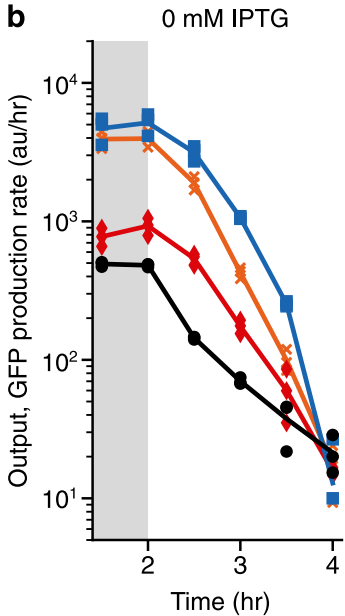

$10 \mathrm{mM} \mathrm{IPTG}$

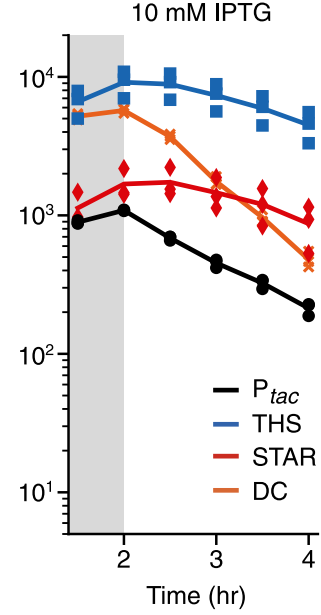

Fig. 5 Performance of single- and multi-level controllers in a cell-free expression system. a Response functions of the controllers showing output GFP production rates in arbitrary fluorescence units per hour (arb. units/hr) at $4 \mathrm{hr}$ after the start of the cell-free reaction for varying input IPTG concentrations $(0,0.002,0.01,0.05,0.1,0.25,0.5,1,2,5,10 \mathrm{mM})$. Points show the three biological replicates for each controller and condition (black circles, $\mathrm{P}_{\text {taci }}$ blue squares, THS; red diamonds, STAR; orange crosses, DC). b Output GFP production rate of the controllers over time since the start of the reaction. Time courses shown for controllers in an 'off' (0 mM IPTG; left) and 'on' (10 mM IPTG; right) state. GFP production rates at each time point calculated as an average GFP production rate over the previous $1.5 \mathrm{hr}$ (Methods). Shaded region shows time period over which no regulation is observed. Source data are provided as a Source Data file.

systems can be created with greatly reduced leaky expression when in an 'off state, while also maintaining high expression rates once induced. Furthermore, we have shown that multi-level regulation creates a more digital-like switch when transitioning between 'off and 'on' states and suppresses low-level transcriptional noise (Fig. 4), both of which are valuable properties when developing genetic systems for information processing or when highly toxic products or excitable systems act as downstream products.

Our top MLC design, which makes use of a THS for L2 regulation, achieved $>2000$-fold change in output upon induction in vivo and displayed a $10 \mathrm{~dB}$ SNR (Table 1) making it one of the most tightly controlled and high-performance induction systems built to date. Furthermore, the flexibility of our modular genetic toolkit for assembling new multi-level controllers (Fig. 2), and the availability of many other THSs, makes it easy to develop additional orthogonal MLCs that could be used in parallel within the same cell. It is worth noting that the underlying $\mathrm{P}_{t a c}$ promoter that the THS MLC uses achieved only a 93-fold change and 0.2 $\mathrm{dB}$ SNR when used alone as an SLC. Therefore, employing the multi-level regulatory approach outlined in this work could offer a means to greatly improve the performance of many existing low-performance transcriptional sensors, without any need to modify the transcription factors or promoter sequences making up these devices.

With the improvements we see when employing multi-level regulation, it is likely no coincidence that small interfering RNAs (siRNAs) are also widely used by bacteria to refine the regulation of many endogenous processes ${ }^{51-53}$. RNAs are perfectly tailored for this task, imposing a small metabolic burden and offering a fast response. In this work, we selected synthetic RNA-based regulators that function through RNA-RNA hybridisation alone. While this reduces our dependencies on other cellular machinery and makes them easier to transfer between strains/organisms, it is known that many endogenous siRNA regulators employ protein chaperones such as $\mathrm{Hfq}$ to increase their binding affinity to targets and strengthen their regulatory effect ${ }^{54}$. It would be interesting to explore the use of synthetic regulators that make use of these chaperones $^{38}$ or exploit recent advances in the RNA part design ${ }^{16}$ to see whether further improvements in performance are possible.
The stringent regulation of our controllers is achieved by incorporating a C1-FFL regulatory motif that is known to be evolutionarily selected in many natural and engineered systems ${ }^{55}$ and can be used to implement many useful functionalities ${ }^{22}$. More recent work has also demonstrated the importance of interconnections and clustering of many motifs in coordinating more complex behaviours ${ }^{56,57}$. While this work focused on demonstrating that transcriptional and translational regulation can fit neatly into a C1-FFL structure, an intriguing future direction would be to explore how these higher-level structures (e.g. motif clusters or higher-level network structures) might be implemented using the approaches outlined in this work to aid the coordination of multiple interrelated processes in parallel.

This study started with the goal of more stringently controlling gene expression. However, through the design of our MLCs it became evident that the more intricate regulatory designs we built had many other benefits. Synthetic biology to date has often focused on simplifying complexity and reducing systems to their minimal parts. Our findings indicate that complementary studies exploring the complexification of synthetic regulatory systems might also reap rewards allowing us to more efficiently exploit the capabilities of biology by combining many diverse processes and parts in unison. The genetic toolkit presented here offers a starting point for such studies focused on the fundamental processes of transcription and translation.

\section{Methods}

Strains, media and chemicals. All cloning and characterization of genetic constructs was performed using Escherichia coli strain DH10- $\beta$ ( $\Delta$ (ara-leu) 7697 araD139 fhuA $\Delta$ lacX74 galK16 galE15 e14- $\phi 80$ dlacZ $\Delta$ M15 recA1 relA1 endA1 nupG rpsL (StrR) rph spoT1 $\Delta$ (mrr-hsdRMS-mcrBC) (New England Biolabs, C3019I). Cells were grown in DH10- $\beta$ outgrowth medium (New England Biolabs, B9035S) for transformation, LB broth (Sigma-Aldrich, L3522) for general propagation, and M9 minimal media supplemented with glucose $\left(6.78 \mathrm{~g} / \mathrm{L} \mathrm{Na}_{2} \mathrm{HPO}_{4}\right.$, $3 \mathrm{~g} / \mathrm{L} \mathrm{KH}_{2} \mathrm{PO}_{4}, 1 \mathrm{~g} / \mathrm{L} \mathrm{NH}_{4} \mathrm{Cl}, 0.5 \mathrm{~g} / \mathrm{L} \mathrm{NaCl}$ (Sigma-Aldrich, M6030), 0.34 g/L thiamine hydrochloride (Sigma T4625), 0.4\% D-glucose (Sigma-Aldrich, G7528), $0.2 \%$ casamino acids (Acros, AC61204-5000), $2 \mathrm{mM} \mathrm{MgSO}_{4}$ (Acros, 213115000), and $0.1 \mathrm{mM} \mathrm{CaCl}_{2}$ (Sigma-Aldrich, C8106)) for characterization experiments. Antibiotic selection was performed using $100 \mu \mathrm{g} / \mathrm{mL}$ ampicillin (Sigma-Aldrich, $\mathrm{A} 9518$ ) and $50 \mu \mathrm{g} / \mathrm{mL}$ kanamycin (Sigma-Aldrich, K1637). Induction of the expression systems was performed using varying concentrations of isopropyl $\beta$-D1-thiogalactopyranoside (IPTG) (Sigma-Aldrich, I6758). 
Assembly of controllers. All part plasmids were either directly synthesised (GeneArt, Thermo Fisher Scientific) or assembled as complementary singlestranded DNA oligos annealed together. Controllers consisting of 8 parts $(\mathrm{pA}-\mathrm{pH})$ plus a backbone (pMLC-BB1) were assembled using a standard Golden Gate cloning method (Fig. 2B) ${ }^{27}$. Briefly, for each assembly, we started with $18.5 \mathrm{ng}$ of required part plasmids $(\mathrm{pA}-\mathrm{pH})$ and $18.5 \mathrm{ng}$ of the backbone (pMLC-BB1) to be added to a $5 \mu \mathrm{L}$ Golden Gate reaction. The standard manufacturer's reaction conditions were used, but at a quarter of their normal volume (New England Biolabs, E1601). Two microliters of this reaction mix was then used to transform $12.5 \mu \mathrm{L}$ of chemically competent DH10- $\beta$ cells (New England Biolabs, C3019). All assembled constructs were sequence verified by Sanger sequencing (Eurofins Genomics). Annotated sequences of all part and backbone plasmids and assembled controllers are provided in GenBank format in Supplementary Data 2. Plasmid maps are shown in Supplementary Figs 4 and 5. All plasmids are available from Addgene (\#166922-166941).

Characterisation experiments. Single colonies of cells transformed with an appropriate genetic construct were inoculated in $200 \mu \mathrm{L}$ M9 media supplemented with glucose and kanamycin for selection in a 96-well microtiter plate (Thermo Fisher Scientific, 249952). Cultures were grown for $14 \mathrm{hr}$ in a shaking incubator (Stuart, S1505) at $37^{\circ} \mathrm{C}$ and $1250 \mathrm{rpm}$. Following this, the cultures were diluted 3:40 $(15 \mu \mathrm{L}$ in $185 \mu \mathrm{L})$ in $\mathrm{M} 9$ media supplemented with glucose, kanamycin for selection and IPTG for induction in a new 96-well microtiter plate and grown for a further $4 \mathrm{hr}$ under the same conditions. Finally, the cultures were further diluted $1: 10(10 \mu \mathrm{L}$ into $90 \mu \mathrm{L})$ in phosphate-buffered saline (PBS) (Gibco,18912-014) containing $2 \mathrm{mg} / \mathrm{mL}$ kanamycin to halt protein translation. These samples were incubated at room temperature for $1 \mathrm{hr}$ to allow for full maturation of GFP before flow cytometry was performed.

Flow cytometry. Measurements of GFP fluorescence in single cells was performed using an Acea Biosciences NovoCyte 3000 flow cytometer equipped with a NovoSampler to allow for automated collection of samples from a 96-well microtiter plate. Data collection was performed using the NovoExpress version 1.2.4 software. Cells were excited using a $488 \mathrm{~nm}$ laser and GFP fluorescence measurements taken using a $530 \mathrm{~nm}$ detector. At least $10^{6}$ events were captured per sample. In addition, to enable conversion of GFP fluorescence into calibrated MEFL units ${ }^{58}$ a single well per plate contained $15 \mu \mathrm{L}$ of 8-peak Rainbow Calibration Particles (Spherotech, RCP-30-5A) diluted into $200 \mu \mathrm{L}$ PBS. Automated gating of events and conversion of GFP fluorescence into MEFL units was performed using the forward (FSC) and side scatter (SSC) channels and the FlowCal Python package version 1.2 with default parameters ${ }^{58}$. To correct for the GFP autofluorescence of cells, E. coli DH10- $\beta$ cells containing no genetic construct were grown in identical conditions. An average measurement of GFP fluorescence in MEFL units from three biological replicates of these cells was then subtracted from fluorescence measurements of cells containing our genetic constructs to correct for cell autofluorescence.

Plate reader measurements of construct performance in vivo. Single colonies of cells transformed with an appropriate genetic construct were inoculated in $200 \mu \mathrm{L}$ M9 media supplemented with glucose and kanamycin for selection in a 96-well microtiter plate (Thermo Fisher Scientific, 249952). Cultures were grown for $4 \mathrm{hr}$ in a shaking incubator (Stuart, S1505) at $37^{\circ} \mathrm{C}$ and $1250 \mathrm{rpm}$. Following this, the cultures were diluted 3:40 $(15 \mu \mathrm{L}$ in $185 \mu \mathrm{L})$ in $\mathrm{M} 9$ media supplemented with glucose, kanamycin for selection and IPTG for induction in a 96-well $190 \mu \mathrm{m}$ clear base imaging microplate (4titude, Vision Plate ${ }^{\mathrm{TM}}, 4 \mathrm{ti}-0223$ ). This spectrophotometric assay was performed using a BioTek Synergy Neo2 plate reader at $37^{\circ} \mathrm{C}$. Optical density at $600 \mathrm{~nm}\left(\mathrm{OD}_{600}\right)$ was measured every $10 \mathrm{~min}$ over a $16-\mathrm{hr}$ period. $\mathrm{OD}_{600}$ measurements were also taken from samples of $\mathrm{M} 9$ medium supplemented with glucose containing no cells to allow for quantification of media autofluorescence. Samples were shaken continuously throughout the experiment. Data collection was performed using the Gen5 version 3.04 software. For each time point, media autofluorescence was subtracted from the sample measurement.

Cell-free expression. The E. coli cell lysate for CFPS was prepared using an autolysis protocol ${ }^{59}$. In this protocol, E. coli BL21-Gold (DE3) cells harboring a pAS-LyseR plasmid give a high-quality cell lysate by freeze-thawing. Specifically, these cells were grown overnight at $37^{\circ} \mathrm{C}$ in LB broth supplemented with ampicillin. On the following day, cells were subcultured in $2 \mathrm{~L}$ of $2 \times$ YTPG medium supplemented with ampicillin and grown at $37^{\circ} \mathrm{C}$ to an $\mathrm{OD}_{600}$ of 1.5. Cells were then harvested at $2000 \times g$ for $15 \mathrm{~min}$ at room temperature in four centrifuge bottles and $45 \mathrm{~mL}$ of cold S30A buffer $(50 \mathrm{mM}$ Tris- $\mathrm{HCl}$ at $\mathrm{pH} 7.7,60 \mathrm{mM}$ potassium glutamate, $14 \mathrm{mM}$ magnesium glutamate, final $\mathrm{pH}$ 7.7) was added to each. Cells were then resuspended by vigorous vortex mixing and poured into a pre-weighted $50 \mathrm{~mL}$ falcon tube and centrifuged as in the previous step. The supernatants were completely removed, and the falcon tubes weighted again. The net weight of each pellet was calculated and relative to its weight two volumes of cold S30A supplied with $2 \mathrm{mM}$ DTT was added ( $3 \mathrm{~mL}$ for $1.5 \mathrm{~g}$ of pellet). After vigorous vortex mixing, the samples were stored at $-80^{\circ} \mathrm{C}$. The next day, frozen cells were placed in a room temperature water bath to thaw, vigorously vortexed, incubated at $37^{\circ} \mathrm{C}$ on a shaker for $45 \mathrm{~min}$, vortex mixed again, and then incubated at $37^{\circ} \mathrm{C}$ for $45 \mathrm{~min}$ The samples were then centrifuged at $30,000 \times g$ for $60 \mathrm{~min}$ at $4^{\circ} \mathrm{C}$. The supernatants were carefully pipetted out and aliquoted in $1.5 \mu \mathrm{L}$ tubes, and then finally centrifuged at $20,000 \times g$ using a tabletop centrifuge for $5 \mathrm{~min}$ to remove residual cell debris. Aliquots of the lysate were stored at $-80^{\circ} \mathrm{C}$ after flashfreezing in liquid nitrogen.

For the prepared lysate, $\mathrm{Mg}$-glutamate and $\mathrm{K}$-glutamate were titrated in with all components of the cell-free reaction based on the protocol of Sun et al. ${ }^{60}$ resulting in concentrations of $10 \mathrm{nM}$ and $60 \mathrm{mM}$, respectively, for optimal GFP production. Each reaction was prepared at a final volume of $10.5 \mu \mathrm{L}$ containing $33 \%$ lysate, $\mathrm{Mg}$ glutamate and K-glutamate as titrated, and amino acids mix, energy mix, and PEG 8000. For cell-free experiments of the SLC and MLC constructs, maxi-prepped plasmids (using Machery-Nagel NucleoBond Xtra Maxi kit) were added at a final concentration of $10 \mathrm{nM}$ along with varying concentrations of IPTG $(0,0.002,0.01$, $0.05,0.1,0.25,0.5,1,2,5,10 \mathrm{mM})$. While gently mixing by pipette, $10 \mu \mathrm{L}$ of reactions were transferred to a 384-well plate (Greiner Bio-One, 784076) and GFP fluorescence was monitored (excitation/emission wavelengths of $485 / 528 \mathrm{nM}$ and gain $=100$ ) every $10 \mathrm{~min}$ in a plate reader (Tecan Infinite $200 \mathrm{PRO}$ ).

Signal to noise ratio. The signal to noise ratio (SNR) in decibel (dB) units was calculated from the flow cytometry GFP fluorescence distributions using the equation ${ }^{23}$

$$
\mathrm{SNR}_{\mathrm{dB}}=20 \cdot \log _{10} \frac{\left|\log _{10}\left(\mu_{\mathrm{ON}} / \mu_{\mathrm{OFF}}\right)\right|}{2 \cdot \log _{10}(\sigma)} .
$$

Here, $\mu_{\mathrm{ON}}$ and $\mu_{\mathrm{OFF}}$ are the geometric means of distributions for the 'on' and 'off states, respectively, and $\sigma$ is the geometric standard deviation of the distribution for the 'off state. 'off and 'on' states correspond to cells grown in 0 $\mathrm{mM}$ and $1 \mathrm{mM}$ IPTG, respectively.

Data analysis and numerical simulation. Data analysis was performed using Python version 3.7.4 and the NumPy version 1.17.4, SciPy version 1.3.1, Pandas version 1.0.3, FlowCal version 1.2, and Matplotlib version 3.1.1 libraries. ODE models were simulated using the odeint function of the SciPy package with default parameters. Steady-state response functions of the controllers were calculated by fitting median GFP fluorescence values from the flow cytometry distributions for a range of input IPTG concentrations to the following Hill function

$$
y=y_{\min }+\left(y_{\max }-y_{\min }\right) \frac{x^{n}}{K^{n}+x^{n}} .
$$

Here, $y$ is the output GFP fluorescence in MEFL units, $y_{\min }$ and $y_{\max }$ are the minimum and maximum output GFP fluorescence in MEFL units, respectively, $K$ is the input IPTG concentration at which the output is half-maximal, $n$ is the Hill coefficient, and $x$ is the input IPTG concentration. Fitting of the experimental data was performed using non-linear least squares and the curve_fit function from the SciPy package. Genetic diagrams were generated using DNAplotlib version $1.0^{61,62}$ and figures were composed using Omnigraffle version 7.15 and Affinity Designer version 1.8.3.

Reporting summary. Further information on research design is available in the Nature Research Reporting Summary linked to this article.

\section{Data availability}

Annotated sequences for all plasmids in GenBank format are available in Supplementary Data 2. Flow cytometry data from this study are available at: https://osf.io/wm9cq/ Any other relevant data are available from the authors upon reasonable request. Source data are provided with this paper.

\section{Code availability}

Python scripts for simulating the ODE models of the direct and multi-level controllers can be found in Supplementary Data 1 .

Received: 26 August 2020; Accepted: 18 February 2021; Published online: 19 March 2021

\section{References}

1. de Boer, H. A., Comstock, L. J. \& Vasser, M. The tac promoter: a functional hybrid derived from the trp and lac promoters. Proc. Natl Acad. Sci. USA 80, 21 (1983).

2. Gallivan, J. P. Toward reprogramming bacteria with small molecules and RNA. Curr. Opin. Chem. Biol. 11, 612-619 (2007).

3. Baumschlager, A., Aoki, S. K. \& Khammash, M. Dynamic blue light-inducible T7 RNA polymerases (Opto-T7RNAPs) for precise spatiotemporal gene expression control. ACS Synth. Biol. 6, 2157-2167 (2017). 
4. Castillo-Hair, S. M., Baerman, E. A., Fujita, M., Igoshin, O. A. \& Tabor, J. J. Optogenetic control of Bacillus subtilis gene expression. Nat. Commun. 10, 3099 (2019).

5. Sen, S., Apurva, D., Satija, R., Siegal, D. \& Murray, R. M. Design of a toolbox of RNA thermometers. ACS Synth. Biol. 6, 1461-1470 (2017).

6. Sivashanmugam, A. et al. Practical protocols for production of very high yields of recombinant proteins using Escherichia coli. Protein Sci. 18, 936-948 (2009).

7. Olson, E. J., Hartsough, L. A., Landry, B. P., Shroff, R. \& Tabor, J. J. Characterizing bacterial gene circuit dynamics with optically programmed gene expression signals. Nat. Methods 11, 449-455 (2014).

8. Elowitz, M. B., Levine, A. J., Siggia, E. D. \& Swain, P. S. Stochastic gene expression in a single cell. Science 297, 1183 (2002).

9. Raj, A. \& van Oudenaarden, A. Nature, nurture, or chance: stochastic gene expression and its consequences. Cell 135, 216-226 (2008).

10. Rosano, G. L. \& Ceccarelli, E. A. Recombinant protein expression in Escherichia coli: advances and challenges. Front. Microbiol. 5, 172 (2014).

11. Süel, G. M., Garcia-Ojalvo, J., Liberman, L. M. \& Elowitz, M. B. An excitable gene regulatory circuit induces transient cellular differentiation. Nature 440, 545-550 (2006).

12. Khalil, A. S. et al. A synthetic biology framework for programming eukaryotic transcription functions. Cell 150, 647-658 (2012).

13. Deng, D., Yan, C., Wu, J., Pan, X. \& Yan, N. Revisiting the TALE repeat. Protein Cell 5, 297-306 (2014).

14. Gilbert, L. A. et al. Genome-scale CRISPR-mediated control of gene repression and activation. Cell 159, 647-661 (2014).

15. Bartoli, V., Meaker, G. A., di Bernardo, M. \& Gorochowski, T. E. Tunable genetic devices through simultaneous control of transcription and translation. Nat. Commun. 11, 2095 (2020)

16. Chappell, J., Westbrook, A., Verosloff, M. \& Lucks, J. B. Computational design of small transcription activating RNAs for versatile and dynamic gene regulation. Nat. Commun. 8, 1051 (2017).

17. Green, A. A., Silver, P. A., Collins, J. J. \& Yin, P. Toehold switches: de-novodesigned regulators of gene expression. Cell 159, 925-939 (2014).

18. Cameron, D. E. \& Collins, J. J. Tunable protein degradation in bacteria. Nat. Biotechnol. 32, 1276-1281 (2014).

19. Meyer, A. J., Segall-Shapiro, T. H., Glassey, E., Zhang, J. \& Voigt, C. A. Escherichia coli "Marionette" strains with 12 highly optimized small-molecule sensors. Nat. Chem. Biol. 15, 196-204 (2019).

20. Calles, B., Goñi-Moreno, Á. \& de Lorenzo, V. Digitalizing heterologous gene expression in Gram-negative bacteria with a portable ON/OFF module. Mol. Syst. Biol. 15, e8777 (2019).

21. Westbrook, A. M. \& Lucks, J. B. Achieving large dynamic range control of gene expression with a compact RNA transcription-translation regulator. Nucleic Acids Res. 45, 5614-5624 (2017).

22. Mangan, S. \& Alon, U. Structure and function of the feed-forward loop network motif. Proc. Natl Acad. Sci. USA 100, 11980 (2003).

23. Beal, J. Signal-to-noise ratio measures efficacy of biological computing devices and circuits. Front. Bioeng. Biotechnol. 3, 93 (2015).

24. Nielsen, A. A. K. et al. Genetic circuit design automation. Science 352, aac7341 (2016).

25. Golding, I., Paulsson, J., Zawilski, S. M. \& Cox, E. C. Real-time kinetics of gene activity in individual bacteria. Cell 123, 1025-1036 (2005).

26. Woodruff, L. B. A. et al. Registry in a tube: multiplexed pools of retrievable parts for genetic design space exploration. Nucleic Acids Res. 45, 1553-1565 (2016).

27. Engler, C., Gruetzner, R., Kandzia, R. \& Marillonnet, S. Golden gate shuffling: a one-pot DNA shuffling method based on type IIs restriction enzymes. PLoS ONE 4, e5553 (2009).

28. Green, A. A. et al. Complex cellular logic computation using ribocomputing devices. Nature 548, 117 (2017).

29. Chappell, J., Takahashi, M. K. \& Lucks, J. B. Creating small transcription activating RNAs. Nat. Chem. Biol. 11, 214-220 (2015).

30. Zong, Y. et al. Insulated transcriptional elements enable precise design of genetic circuits. Nat. Commun. 8, 52 (2017).

31. Boo, A., Ellis, T. \& Stan, G.-B. Host-aware synthetic biology. Synth. Biol. 14, 66-72 (2019).

32. Ceroni, F., Algar, R., Stan, G.-B. \& Ellis, T. Quantifying cellular capacity identifies gene expression designs with reduced burden. Nat. Methods 12, 415-418 (2015).

33. Ceroni, F. et al. Burden-driven feedback control of gene expression. Nat. Methods 15, 387-393 (2018).

34. Gorochowski, T. E., van den Berg, E., Kerkman, R., Roubos, J. A. \& Bovenberg, R. A. L. Using synthetic biological parts and microbioreactors to explore the protein expression characteristics of Escherichia coli. ACS Synth. Biol. 3, 129-139 (2014).

35. Gorochowski, T. E., Avcilar-Kucukgoze, I., Bovenberg, R. A. L., Roubos, J. A. \& Ignatova, Z. A minimal model of ribosome allocation dynamics captures trade-offs in expression between endogenous and synthetic genes. ACS Synth. Biol. 5, 710-720 (2016).

36. Gyorgy, A. et al. Isocost lines describe the cellular economy of genetic circuits. Biophys. J. 109, 639-646 (2015).

37. Weiße, A. Y., Oyarzún, D. A., Danos, V. \& Swain, P. S. Mechanistic links between cellular trade-offs, gene expression, and growth. Proc. Natl Acad. Sci. USA 112, E1038 (2015).

38. Kelly, C. L. et al. Synthetic negative feedback circuits using engineered small RNAs. Nucleic Acids Res. 46, 9875-9889 (2018).

39. Malakar, P. \& Venkatesh, K. V. Effect of substrate and IPTG concentrations on the burden to growth of Escherichia coli on glycerol due to the expression of Lac proteins. Appl. Microbiol. Biotechnol. 93, 2543-2549 (2012).

40. Gomes, L., Monteiro, G. \& Mergulhão, F. The impact of IPTG induction on plasmid stability and heterologous protein expression by Escherichia coli biofilms. Int. J. Mol. Sci. 21, 576 (2020).

41. Perez, J. G., Stark, J. C. \& Jewett, M. C. Cell-free synthetic biology: engineering beyond the cell. Cold Spring Harb. Perspect. Biol. 8, a023853 (2016).

42. Niederholtmeyer, $\mathrm{H}$. et al. Rapid cell-free forward engineering of novel genetic ring oscillators. eLife 4, e09771 (2015).

43. Karim, A. S. \& Jewett, M. C. A cell-free framework for rapid biosynthetic pathway prototyping and enzyme discovery. Metab. Eng. 36, 116-126 (2016)

44. Kelwick, R. J. R., Webb, A. J. \& Freemont, P. S. Biological materials: the next frontier for cell-free synthetic Biology. Front. Bioeng. Biotechnol. 8, 399 (2020).

45. Laohakunakorn, N. et al. Bottom-up construction of complex biomolecular systems with cell-free synthetic biology. Front. Bioeng. Biotechnol. 8, 213 (2020).

46. Borkowski, O. et al. Cell-free prediction of protein expression costs for growing cells. Nat. Commun. 9, 1457 (2018).

47. Swank, Z., Laohakunakorn, N. \& Maerkl, S. J. Cell-free gene-regulatory network engineering with synthetic transcription factors. Proc. Natl Acad. Sci. USA 116, 5892 (2019).

48. Pandi, A., Grigoras, I., Borkowski, O. \& Faulon, J.-L. Optimizing cell-free biosensors to monitor enzymatic production. ACS Synth. Biol. 8, 1952-1957 (2019).

49. McGuffee, S. R. \& Elcock, A. H. Diffusion, crowding \& protein stability in a dynamic molecular model of the bacterial cytoplasm. PLoS Comput. Biol. 6, e1000694 (2010).

50. Niederholtmeyer, H., Stepanova, V. \& Maerkl, S. J. Implementation of cell-free biological networks at steady state. Proc. Natl Acad. Sci. USA 110, 15985 (2013).

51. Gottesman, S. Micros for microbes: non-coding regulatory RNAs in bacteria. Trends Genet. 21, 399-404 (2005).

52. Storz, G., Opdyke, J. A. \& Zhang, A. Controlling mRNA stability and translation with small, noncoding RNAs. Curr. Opin. Microbiol. 7, 140-144 (2004).

53. Waters, L. S. \& Storz, G. Regulatory RNAs in bacteria. Cell 136, 615-628 (2009).

54. Soper, T., Mandin, P., Majdalani, N., Gottesman, S. \& Woodson, S. A. Positive regulation by small RNAs and the role of Hfq. Proc. Natl Acad. Sci. USA 107, 9602 (2010)

55. Milo, R. et al. Network motifs: simple building blocks of complex networks. Science 298, 824 (2002).

56. Gorochowski, T. E., Grierson, C. S. \& di Bernardo, M. Organization of feedforward loop motifs reveals architectural principles in natural and engineered networks. Sci. Adv. 4, eaap9751 (2018).

57. S. Dunn, H. Kugler \& B. Yordanov. Formal analysis of network motifs links structure to function in biological programs. IEEE/ACM Trans. Comput. Biol. Bioinformatics https://doi.org/10.1109/TCBB.2019.2948157 (2019).

58. Castillo-Hair, S. M. et al. FlowCal: A user-friendly, open source software tool for automatically converting flow cytometry data from arbitrary to calibrated units. ACS Synth. Biol. 5, 774-780 (2016).

59. Didovyk, A., Tonooka, T., Tsimring, L. \& Hasty, J. Rapid and scalable preparation of bacterial lysates for cell-free gene expression. ACS Synth. Biol. 6, 2198-2208 (2017).

60. AU - Sun, Z. Z. et al. Protocols for implementing an Escherichia coli based TX-TL cell-free expression system for synthetic biology. J. Vis. Exp. https:// doi.org/10.3791/50762 (2013).

61. Bartoli, V., Dixon, D. O. R. \& Gorochowski, T. E. in Synthetic Biology: Methods and Protocols (ed. Braman, J. C.) 399-409 (Springer New York, 2018).

62. Der, B. S. et al. DNAplotlib: Programmable visualization of genetic designs and associated data. ACS Synth. Biol. 6, 1115-1119 (2017).

\section{Acknowledgements}

We wish to acknowledge the assistance of Dr. Andrew Herman and the University of Bristol Faculty of Biomedical Sciences Flow Cytometry Facility. This work was supported by BrisSynBio, a BBSRC/EPSRC Synthetic Biology Research Centre grant BB/L01386X/1 (T.E.G., C.S.G.), a Royal Society PhD Studentship (F.V.G.), a Royal Society University Research Fellowship grant UF160357 (T.E.G.), the Max Planck Society (T.J.E.), and a 
European Molecular Biology Organization (EMBO) long-term postdoctoral fellowship (A.P.).

\section{Author contributions}

T.E.G. conceived the study. F.V.G. designed the genetic toolkit, assembled all controllers and performed the in vivo experiments. A.P. performed the in vitro cell-free experiments. T.E.G. developed and simulated the mathematical models. F.V.G. and T.E.G. analysed the data. T.E.G., C.S.G. and T.J.E. supervised the work. F.V.G., T.E.G. and C.S.G. wrote the manuscript with input from all other authors.

\section{Competing interests}

The authors declare no competing interests.

\section{Additional information}

Supplementary information The online version contains supplementary material available at https://doi.org/10.1038/s41467-021-21995-7.

Correspondence and requests for materials should be addressed to T.E.G.

Peer review information Nature Communications thanks the anonymous reviewer(s) for their contribution to the peer review of this work.
Reprints and permission information is available at http://www.nature.com/reprints

Publisher's note Springer Nature remains neutral with regard to jurisdictional claims in published maps and institutional affiliations.

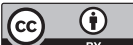

Open Access This article is licensed under a Creative Commons Attribution 4.0 International License, which permits use, sharing, adaptation, distribution and reproduction in any medium or format, as long as you give appropriate credit to the original author(s) and the source, provide a link to the Creative Commons license, and indicate if changes were made. The images or other third party material in this article are included in the article's Creative Commons license, unless indicated otherwise in a credit line to the material. If material is not included in the article's Creative Commons license and your intended use is not permitted by statutory regulation or exceeds the permitted use, you will need to obtain permission directly from the copyright holder. To view a copy of this license, visit http://creativecommons.org/ licenses/by/4.0/.

(C) The Author(s) 2021 